\title{
Nursing in the time of COVID-19: exploring nurse preparedness early in a global pandemic
}

\author{
AUTHORS \\ GED WILLIAMS RN, BN, Crit. Care Cert., LLM, MHA, \\ FACN, FACHSM, FAAN ${ }^{1}$ \\ ANASTASIA GUNN RN, BN ${ }^{2}$ \\ AMY SWEENY RN BSC MPH ${ }^{3,4}$
}

\section{CORRESPONDING AUTHOR}

GED WILLIAMS Griffith University, Australia, Area Director of Nursing \& Midwifery, South Metropolitan Health Service, Perth Australia 6000. Email: ged_williams@hotmail.com
1 School of Nursing and Midwifery, Griffith University, Queensland, Australia

2 Post Anaesthetic Care Unit, Murwillumbah District Hospital, Murwillumbah, New South Wales, Australia.

3 Emergency Department, Gold Coast Hospital and Health Service, Queensland, Australia.

4 School of Medicine, Griffith University, Queensland, Australia.

\section{ABSTRACT \\ Objective: To obtain Australian nurses' perspectives regarding COVID-19 preparedness early in the pandemic. \\ Background: In March 2020 Australia experienced a rise in COVID-19 cases and was bracing itself for the worst, having witnessed China, Europe and America's earlier exposure to the outbreak. On 14 March 2020 an Australian nurse set up a Facebook page called "Nursing in the time of COVID-19: A clinical forum" allowing colleagues to share information about COVID-19. This presented an ideal opportunity to survey nurses' preparedness for the pandemic.}

Study design and methods: Anonymous web-based survey disseminated between 9 April-4 May 2020 via the Facebook page.

Results: A total of 214 nurses responded to the survey of whom $85 \%$ were direct care clinical staff and 29 (13.5\%) had cared for a COVID-19 patient. Prevalent feelings regarding COVID-19 were anxious, overwhelmed, vulnerable, engaged and interested. The strongest sources of information regarding COVID-19 were published academic articles, WHO/ CDC and similar authorities, employer leaflets/ guidance and Facebook. The strongest needs/ expectations identified by the respondents were education and information, access to mental health counselling, provision of additional health workers to support nurses, and supplemental pay rise. Respondents identified work-related training specific to COVID-19, revision of skills and competencies and upskilling to do tasks previously not familiar to the participant of particular benefit. Nurses who had cared for COVID-19 patients expressed a stronger interest in receiving a supplemental pay rise for risk exposure, a stronger sense that they and their employer were ready to care for COVID-19 patients yet expressed less appreciation for mental health counselling compared to nurses who had not yet cared for COVID-19 patients.

Discussion: The perspectives of Australian nurses are generally similar to nurses in other countries. However, we report some variations between nurses who have and have not cared for COVID-19 patients. We identify the levels of concern as well as those supportive actions that are most appreciated by nurses responding to COVID-19 specifically, these same findings could be applied to other major events generally. 
Conclusion: The timing of this survey allowed us to explore the perspectives of Australian nurses during the early pandemic vulnerability period without the benefit of hindsight. Despite thinking the Australian experience was going to be worse than it was, Australian nurses showed relative confidence in their own and their employers' readiness and this was most obvious in nurses who had cared for COVID-19 patients.

\section{What is already known about the topic?}

- Nurses around the world have voiced anxiety and concern about the COVID-19 pandemic and the impact it may have on them personally and professionally.

- Nurses have access to informal networks, social media, media, employer guidelines and other professional and academic sources of information to stay informed and up-to-date with current information on COVID-19 that may help to allay their concerns.

\section{What this paper adds}

- The perspectives of Australian nurses early in the development of the COVID-19 pandemic in relation to how prepared they felt at the time and where they were obtaining information to help guide their thinking and their practice.

- A synopsis of the role and benefits of a social media platform to allow nurses to share thoughts, articles of interest and experiences of COVID-19.

- Opinions vary based on whether a nurse had already cared for a COVID-19 patient or not.

Keywords: COVID-19, nurse, social media, disaster preparedness, pandemic

\section{INTRODUCTION}

The emergence of COVID-19 (also known as Coronavirus and SARS-CoV-2) cases in Australia in late January 2020 captured mainstream media and social media attention and forced all health authorities to move quickly to prepare for what could be a major outbreak of the disease in the context of a global pandemic. Experiences from China, Europe and America indicated Australian health authorities and professionals should prepare to deal with a rapidly spreading and very deadly form of Coronavirus. A major concern was the limited clinical and scientific experience and evidence to inform exactly how this disease might play out in Australia.

Despite the best efforts of government officials, scientists and health experts to inform the community of the situation, the lack of experience and knowledge of this particular virus strain caused fear, anxiety and panic in some parts of the Australian community. ${ }^{1}$ By the second week of March, Lifeline (a crisis-support and suicide prevention phone service in Australia) were receiving 3,00o calls per day of which $23 \%$ were COVID-19 related (normal call rate is about 2,200 per day). ${ }^{2}$

Healthcare professions were also unsure of how they would need to act and react to the emerging scenario in Australia, prompting the Nursing and Midwifery Board of Australia to issue an announcement on 12 March 2020 stating that: The NMBA recognises that as health practitioners, you may be feeling anxious and concerned about decisions you may need to take to provide the best care in these challenging and uncertain circumstances, especially if the coronavirus becomes more widespread. ${ }^{3}$ The Australian Nursing and Midwifery Federation (ANMF) also presented media releases challenging government and employer approaches to, among other things, manpower decisions, including the use of newly graduate nurses and students. ${ }^{4}$ These were unprecedented times with unprecedented announcements and responses throughout the healthcare community. The level of uncertainty and lack of clear guidance and occasionally incorrect information from some authorities in the early phases caused concern for many nurses and other healthcare providers as the numbers of confirmed cases and deaths started to mount throughout the month of March.

Cognisant of this concern, a variety of online and social media-based forums were established to provide access to information, and an opportunity for discussion and interaction, as well as an avenue to address the escalating professional and social changes presented by the spread of COVID-19. A Facebook page was established on 14 March 2020 by one of the authors of this article (AG) for her nursing and midwifery colleagues to start sharing their experiences and information they could find on the disease: Nursing in the time of COVID-19: A clinical forum (AGsFP). Within one month the page had attracted 37,00o contacts worldwide, mostly in Australia. ${ }^{5}$

In order to better understand why so many nurses (and midwives) requested to join this FB page, a brief online questionnaire was developed to explore their perspectives and concerns with the unfolding COVID-19 scenario. The purpose of the survey was to find out what resources and support mechanisms were working for them and what else could be done to support them and finally, how the page was assisting them in their understanding of the disease and consequences for them as nurses. 


\section{METHOD}

An online survey tool was developed to explore the perspectives and concerns that AGsFP users were facing. The survey was designed by the authors and reviewed by two academics experienced in survey design and face validation. Iteratively the survey was refined to capture the information required from nurses, the emerging and final findings were discussed, and consensus derived. A previously validated tool was not sought as this was a relatively unique study and audience, however questions asked were similar to many exploratory surveys of nurses in other contexts. Questions were all written in a positive manner to make Likert test scoring and analysis easy. A purposive convenience sample was used by providing an open invitation to members of the AGsFP to participate. Two separate reminder notices were sent to try and maximise participation. Participants were encouraged to ask colleagues to join the survey pool in a quasi-snowballing approach.

The final survey comprised six sections. Section one asked general questions about participant characteristics including age, gender, years in nursing, the speciality area of practice and the setting of practice. Sections 2-6 used Likert scales 1-5 where $1=$ Strongly Disagree, $2=$ Disagree, $3=$ Neutral, 4=Agree, $5=$ =Strongly Agree. Section 2 asked about their domestic and professional setting. Respondents were asked to indicate how supportive and understanding the other people in their household were towards their professional responsibilities at work and how their work colleagues were towards their personal responsibilities at home. Section 3 required respondents to indicate their agreement with statements about their general wellbeing, feelings and experiences during this time of COVID-19 compared to before the outbreak. Section 4 required respondents to indicate their agreement with statements about how helpful various sources of information, training, education and other resources regarding COVID-19 had been. Section 5 asked participants to indicate how ready they thought they were to care for COVID-19 patients and also how ready they thought their employer, community and country were. In section 6 participants were asked to indicate their agreement with various statements about the FB page, and to estimate the final positive cases and deaths they expected from COVID-19 in Australia (so as to gauge their sense of optimism or pessimism with the impending pandemic).

The authors provided a detailed cover letter informing potential participants of the purpose and nature of the study and clarified that ethics approval had not been sought. We further explained that all responses would not be identifiable, and results aggregated and published. Knowing the emotional situation for some nurses involved in the pandemic response we made the following important statement: "If at any time during the filling out of the survey you have negative feelings, please stop. Go for a walk or do something else for a while. Come back and complete it if you feel up to it, but if not, no problem. Your wellbeing is our priority, so don't feel bad if you do not wish to participate".

The link to the final survey was distributed via the Facebook page to all members of the group; participation was both anonymous and voluntary. The survey was released on 9 April and closed on 4 May.

\section{DATA ANALYSIS}

A description of participants was produced using simple counts and proportions. For Likert questions, the number of respondents was tallied for each question, and the proportion responding to each response was calculated. A score was assigned to each response with values ranging from 5 (strongly agree) to 1 (strongly disagree). An overall average weighted score was created by multiplying the number of respondents for each response by the response value, then summing these products, and then dividing by the total number of respondents for that question. Free text statements were collated, themed and summarised by two authors. Comparisons of the proportion of respondents agreeing or strongly agreeing with each statement were made between nurses who had cared for a COVID-19 patient and those that had not using the Fisher's exact test and the statistical program Python. To avoid multiple-testing issues, only the questions or statements that were most discrepant between the two groups were tested for statistical significance. A p-value of <0.05 was considered statistically significant.

\section{RESULTS}

A total of 214 nurses eligible to practice nursing in Australia responded, of which 90\% $(n=192)$ were female and $86 \%$ were direct care clinical staff. The average age of nurse respondents was 48.6 years, with only six nurses (3.0\%) aged less than 30 years old. More than $85 \%$ of responses were received in the first 10 days of the survey period. Respondents had been aware of the threat of COVID-19 for an average of 67 days at the time of the survey. At the time of surveying only 29 respondents (13.5\%) had cared for a COVID-19 patient, of which 55\% worked in Critical Care or Emergency areas. The remaining characteristics of participants are shown in Table 1 (Supplementary files).

Twenty-four nurses did not answer any of the statement questions. Of these, three had cared for COVID-19 patients.

The distribution of responses to each survey question, weighted averages, and medians and interquartile ranges are available in Table 2 (Supplementary files).

Overall nurses perceived people at home to be slightly more understanding of their work responsibilities (Question 1, 2, 3 average score $4.42,4.03,2.25$ ) compared to work colleagues understanding of their family responsibilities (Question 4, 5 , 6 average score $3.83,3.72,2.52$ ). 
Feelings and experiences towards the COVID-19 situation as perceived by the participants when they completed the survey were mixed. Rank ordered from most to least using a five-point Likert scale (where $1=$ strongly disagree and 5 = strongly agree) were anxious (3.85), overwhelmed (3.78), vulnerable (3.76), engaged and interested (3.76), frightened (3.56), difficult to sleep or to stay asleep (3.48), exhausted (3.4), morbidly curious (3.35), positively challenged (3.33), angry (3.13), and relaxed (2.35).

Venues participants use for information regarding COVID-19 at the time of the survey using the same five-point Likert scale were: published academic articles (4.14), World Health Organization (WHO)/ Center for Disease Control (CDC) and similar authorities (3.99), employer leaflets and guidance (3.69), Facebook (3.58), radio news (3.47), TV news (3.45), Google (3.25), word of mouth (3.22), other social media (3.18).

Work-related training specific to COVID-19 (3.69) and revision of skills and competencies (3.69) were considered equally valuable by the respondents followed closely by upskilling to do tasks previously not familiar to the participant (3.59), while employer sponsored mental health lectures/support (2.72) received the least recognition of the options provided (Table 2, Supplementary file).

Participant's perception of readiness for COVID-19 in Australia was further explored. The respondents thought Australia as a country was somewhat ready (3.02), their employer was next most ready (2.72), the participants themselves (2.42) were next and the community was least ready (2.39). However, 65\% (17/26) of those who had cared for COVID-19 patients stated personal readiness compared to $47.2 \%$ (67/157, $\mathrm{p}=0.53)$ of those who had not yet cared for COVID-19 patients (Table 3, Supplementary files).

Participant's perceptions of supports most valuable to them identified that timely education and information was the most important (4.24) followed closely by access to mental health counselling (4.02) and provision of additional health workers to support nurses (4.01). A supplemental pay rise was supported but came fourth among the options provided (3.79). At the time access to PPE had not been raised as a concern in Australia and was omitted as an option.

Participants who had cared for COVID-19 patients identified a supplemental pay rise as 4.2 compared to those who had not 3.73 (Table 3, Supplementary files). Also, those who had cared for COVID-19 patients gave a combined score for access to mental health counselling and support as 3.85 , compared with those who had not cared for COVID-19 patients 4.05 (Table 3, Supplementary files).

Finally, comments towards the provision of AGsFP are summarised at the end of Table 2 (Supplementary files). At the time of the survey AGsFP had approximately 38,000 members. Of the participants who responded $82 \%$ agreed or strongly agreed that AGsFP was useful, $85 \%$ agreed or strongly agreed that the site helped them realise they were not alone in their thoughts and feelings as a nurse, that the site provided messages from other nurses that were reassuring, that it was an essential resource and for many it also provided entertaining relief!

Table 3 (Supplementary files) shows the differences in responses by whether the participant had already cared for a COVID-19 patient or not. Although none of the proportions differed to a statistically significant amount, nurses who had already cared for a COVID-19 patient more frequently agreed that they (65.4\% vs 42.7\%, $\mathrm{p}=0.052)$, and their hospitals ( $65.4 \%$ vs 50.3\%) were ready to care for a large number of COVID-19 patients. However, they were less likely to agree with their community being ready (26.9\% vs $41.4 \%$ ).

In a final question we asked participants to estimate the number of confirmed cases of COVID-19 in Australia and the number of COVID-19 related deaths by the end of 2020 . The median number of confirmed cases estimated by the participants was 20,000 (Range: $100-5,000,000$ ) and the estimated median number of deaths was 929 (Range 8 -150,000). Of the 17 nurses who disclosed the number of COVID-19 patients they had cared for at the time of the survey they averaged six each (Range 1-30).

One hundred and seventy-five (175) nurses provided an open-ended suggestion on the FB site. The two main themes in the comments section were 1. Encouragement to continue to provide relevant and supportive information on the FB site (no change, $\mathrm{n}=100(57.1 \%)$ ) and 2 . Filter and remove any comments or articles that are not evidence based or are unprofessional ( $n=13$, (eg. "Debunk fake news", "Effective filtering of misinformation"). Of the remaining, recurring responses included: provide links to clinical resources ( $n=8)$ (eg. "Post sites with free CPD for up-skilling", and "what nurses can legally do if they do not have the appropriate PPE”), provide links to educational resources $(n=10)$ (eg. "More scientific paper links”, “COVID case studies of various degrees of the disease"), provide links to mental health resources $(n=5)$ (eg. "links to mental health services, counsellors for the people with ongoing issues"), and provide local and/or rural-specific information $(n=9)$. Seven respondents found the site overwhelming or anxietyinducing and two recommended adding more comedy.

\section{DISCUSSION:}

This was a very early study of nurses at the beginning of the Australian pandemic experience and lacked the opportunity to refer to other published surveys relevant to COVID-19 in Australia. At the time of writing (June 2020), Australia had a little over 7,00o confirmed cases of COVID and 10o deaths associated with the disease, significantly less than most developed countries of the world. Australia had reached its peak active COVID-19 cases of almost 5,000 on 4 April and the survey was released five days later on 9 April $2020 .{ }^{6}$ The participants were asked to estimate the confirmed 
COVID-19 cases and deaths by the end of 2020. Based on data as of 26 May 2020, they had over-estimated confirmed cases ( median estimate $=20,000$, actual 7,100) and deaths (median estimate 929, actual 102). However following revision of this manuscript at 31 December 2020, and the impact of a second deadly outbreak of COVID-19 in the state of Victoria, the total case number was 28,408 and the death count 909..... very close to the median estimates of the participants back in April!

Nurses comprise the largest portion of the Australian healthcare workforce. ${ }^{7}$ Studies of nurses personal and professional wellbeing have shown that they are a resilient workforce, capable of coping in a variety of stressful and chaotic situations, while undertaking shift work. ${ }^{8,9}$ Many of the feelings expressed by Australian nurses coming into the COVID-19 pandemic were very similar to those expressed by nurses in other surveys around the world. A survey of 3,500 UK nurses conducted by Nursing Times showed that compared with before the start of the COVID-19 pandemic 33\% now felt a little more anxious and 55\% felt a lot more anxious; and when asked to describe their mental health right now $26 \%$ stated bad and $7 \%$ stated very bad. ${ }^{10}$ A similar study conducted by the Royal College of Nursing (UK) found that of the 2,6oo nurse respondents $33 \%$ reported severe or extremely severe depression, anxiety or stress and $74 \%$ feel their personal health is at risk..$^{11}$ Feelings of anxiety and being overwhelmed were strong in our findings whilst concurrently feeling engaged and interested at the stage we had surveyed the group.

In a survey conducted by National Nurses United (Union) in the US from 15 April to 10 May 2020, 23,000 nurses responded. Of the responding nurses who had provided care to confirmed COVID-19 patients 27\% reported having been exposed without the appropriate PPE and 33\% reported that their employer required them to use their own sick leave, vacation, or paid time off if they contracted COVID-19 or were exposed to COVID-19 and needed to self-quarantine. ${ }^{12}$ In a similar study of critical care practitioners in the US in late March 2020, over 50\% had already cared for COVID-19 patients and $65 \%$ identified their ICU facility was inadequately prepared to care for COVID-19 patients. Further, 94\% anticipated PPE shortages, 59\% anticipated staff shortages, while $54 \%$ stated a lack of clinical guidance/treatment protocols as a concern and $33 \%$ stated concerns receiving pay/benefits during periods of quarantine. ${ }^{13}$ By contrast the participants in this survey gave a sense of reasonable readiness at the individual and employer level. However, what was notable in the Australian context was the differing opinions from those nurses who had cared for COVID-19 patients and those who had not. Those who had cared for COVID-19 patients were slightly more inclined to believe they and their employer were ready for COVID-19 than nurses who had not yet cared for COVID-19 patients. It is postulated that once nurses care for a COVID-19 patient, their confidence may increase compared with those who have not. Also, those with
COVID-19 patient exposure were less inclined to call for access to mental health support (at this stage) but more inclined to want a supplemental payment for risk/stress exposure. Interestingly the French Prime Minister announced that front line healthcare workers would receive a one-off financial bonus and a "significant" pay rise. ${ }^{14,15}$

The Australian participants identified work-related training specific to COVID-19 and revision of skills and competencies were equally valuable followed closely by upskilling to do tasks previously not familiar to the participant. By contrast the RCN study of UK nurses found that $62 \%$ said their redeployment training was either non-existent or inadequate and $52 \%$ said they lacked confidence about COVID-19 infection control or had received no training. ${ }^{9}$ Early training and preparation for new skills or refreshing knowledge of skills pertinent to the COVID-19 situation are necessary to give staff the skill and confidence they need to manage in trying situations such as a COVID-19 pandemic.

For years governments and health departments have been asked to prepare for major health emergencies and have witnessed SARS, Ebola and a range of Influenza epidemics triggering the importance of pandemic readiness. ${ }^{16}$ It is therefore troubling that many well-developed health systems were unable to support and prepare their nursing workforce to the level necessary for this pandemic. Training, education, competency and readiness, are all critical to nurses' feelings of confidence and personal wellbeing and can never be under-estimated or ignored. Our study showed nurses preference for published academic and authorised (WHO/ CDC) articles and guidance (Table 2, Supplementary files) however it is also essential that employers guide how such information is to be applied in the context of their health service as this appears to be the area where confusion and concern can arise. It is important that employers are quick to provide clear and consistent instructions that are aligned to the evidence giving nurses the confidence to approach the challenge with sufficient knowledge and skill.

Although sometimes criticised as a less trusted source of news in Australia and the US, ${ }^{17,18}$ we found in this survey that Facebook was a venue where participants in this survey are more likely to source information than other media with respect to COVID-19 and was slightly stronger than radio and TV news services and other social media outlets. However, in the verbatim comments relating to AGsFP participants did ask to remove any comments or articles that are not evidence based or are unprofessional. Studies examining the use of social media as a source for news and information in the US cite Facebook as being four times more prevalent than any other social media platform despite scepticism about content reliability. ${ }^{17}$ In addition to AGsFP, many other professional nursing groups were posting information regarding COVID-19 and directing readers to pertinent and substantial academic and authorised sources. In our subsequent resubmission we have discovered a number 
of studies using Facebook and other social media to better understand the experiences and concerns of the nursing and the broader community. Abuhammad et al. ${ }^{19}$ for example analysed nine Facebook sites in Jordon to discover that nurses in Jordan perceived these sites to be constructive and positive and were supporting and advocating for the sick and their families ${ }^{19}$.

The importance of Facebook sites is to build communities who have a shared interest and need. Our community were nurses interested in knowing more about COVID-19 from a nursing care perspective and were willing to share their opinions, experiences and findings from other sources to this community who were then able to filter and/or pursue such leads to study and share them further. The feedback regarding AGsFP suggests it to be valuable to those who used it and a "passion hobby" for the initiator (AG).

This was a relatively early study of the needs and perceptions of Australian nurses during the COVID-19 outbreak and was fast-tracked to ensure we were able to receive early feedback that could help the AGsFP site and its initiator and also to inform the profession of what nurses were feeling and saying before they had the benefit of hindsight. There were few other studies relevant to COVID-19 in Australia from which to leverage and reference which may have led to gaps in the approach taken.

\section{LIMITATIONS}

Our survey aligns with the timing of the first surveys conducted in the UK and US being late March-early April 2020. Although we did not use previously validated tools (eg. Anxiety scales), we chose to use some similar questions to these other surveys, we also wanted to keep our survey relatively brief to encourage responses. We did not have the benefit of a formal external review process however this did allow us to capture early opinions and thoughts among the Australian nursing community. The low response rate (214 Australian nurses and incomplete responses), possibly associated with the intensity of the ongoing pandemic suggest it is not possible to generalise these results to the population. Nevertheless, the results represent a cross section of Australian nurses which can add to the broader discussion about COVID-19 from a nursing perspective, with a particular emphasis on first impressions as it was unknown at the time of survey how the pandemic would unfold in Australia. Responders were members of AGsFP and likely Facebooksavvy users and may have a bias towards Facebook and other social media sources for their information, although the majority stated that academic and other officially authorised sources and employer leaflets remained a higher order resource than Facebook and other media/social media.

\section{CONCLUSION}

In hindsight we can now see that comparisons between the US and UK and Australian studies of nurses' experiences with COVID-19 are difficult as the UK and US experiences were more widespread, deadly and chaotic compared to the Australian experience. Nevertheless, there are common early findings relevant to nurses on the frontline of this pandemic and potentially other future outbreaks. These include showing and providing support to nurses on the frontline; giving them accurate and authenticated information early in all clinical settings; providing education, training, up skilling/cross training and support to nurses early and ensuring anyone being asked to undertake unfamiliar tasks in unfamiliar environments is given sufficient support to be confident, competent and safe. Acknowledging the important emotional and practical role that family and friends play in supporting those nurses on the frontline and encouraging other nursing colleagues at work to acknowledge and support their colleagues who may have the dual challenge of family and social problems also playing on their mind while at work is important.

We have gained a new appreciation for the value of Facebook sites devoted to specific communities of practice. The Nursing in the time of COVID-19: A clinical forum Facebook site proved to be a frequently accessed resource to help a community of Australian (and other) nurses during a difficult time and an added resource to give many Australian nurses the additional support and guidance they were looking for in the early weeks of the Australian experience of the COVID-19 pandemic.

\section{IMPLICATIONS FOR RESEARCH, POLICY, AND PRACTICE}

- Larger and more detailed studies of the needs and readiness of nurses preparing for major events and pandemics are required.

- Policy makers and healthcare management need to be mindful and inclusive of the perspectives of frontline nurses and their representative organisations to ensure appropriate targeting of resources and training to those nurses in greatest need.

- Nurses need to prepare themselves and their families and friends for inevitable "system shocks" such as pandemics and other major events that will test the profession and the health system generally. Family and friends of nurses play an important role in supporting nurses' during times of professional and work stress. The importance of a nurse's family and friends has been identified in this study and is worthy of further exploration.

- Ensuring contingencies and mitigations are in place to support staffing numbers, equipment requirements, changes in practice and protocols, rapid upskilling and scaling up of human and material resources are vital to avert secondary casualties of such events. 
Acknowledgements: We would like to acknowledge the nurses who took the time to complete this online survey. We also acknowledge the input of Dr Janie Brown and Professor Gavin Leslie of Curtin University who provided academic guidance at the development, design, writing and review stages of the project. We also wish to thank Mary Grace Atienza for managing the survey tool, data collation and analysis.

Conflicts of Interest: The authors have no conflicts of interest to declare.

\section{REFERENCES}

1. Black Dog Institute. Mental health ramifications of COVID-19: the Australian context. Black Dog Institute. 2020. [cited 2020 May 30] Available from: https://www.blackdoginstitute.org.au/ wpcontent/uploads/2020/04/20200319 covid19-evidenceand-reccomendations.pdf

2. Lifeline. Lifeline will continue answering calls through COVID-19 (Media Release: 18 March 2020). Lifeline. 2020. [cited 2020 May 30] Available from: https://www.lifeline.org.au/static/ uploads/files/20200318-lifeline-to-continue-answering-callsthrough-coronavirus-final-wfmgdbkq.pdf

3. Nurses and Midwifery Board of Australia. Statement from the NMBA and AHPRA in response to COVID-19 - 12 March 2020. Nursing and Midwifery Board of Australia. 2020. [cited 2020 May 30] Available from: https://www.nursingmidwiferyboard. gov.au/News/2020-03-12-covid-19.aspx

4. Australian Nursing and Midwifery Federation. Nursing students are only one solution in responding to COVID-19 pandemic (Media Release: 18 March, 2020). Australian Nursing and Midwifery Federation. 2020. [Cited 2020 May 30] Available from: www.anmf.org.au/media-releases/entry/media_200318_2

5. Facebook. Nursing in the time of COVID-19. A clinical forum. Facebook. [cited 2020 May 30] Available from: https://www. facebook.com/groups/608092800035715/

6. Worldometer. Coronavirus: active cases in Australia. Worldometer. 2020 [cited 2020 May 30] Available from: https://www.worldometers.info/coronavirus/country/australia/

7. Australian Institute of Health and Welfare. Australia's health 2018: In brief. Canberra. Australian Institute of Health and Welfare. 2018. [cited 2020 May 30] Available from: https:// www.aihw.gov.au/reports/australias-health/australiashealth-2018/contents/table-of-contents

8. Tahghighi M, Brown JA, Breen LJ, Kane R, Hegney D, Rees CS A comparison of nurse shift workers' and non-shift workers' psychological functioning and resilience. J Adv Nurs. 2019; 75(11): 2570-8. Available from: https://doi.org/10.1111/ jan.14023

9. Cooper AL, Brown JA, Rees CS, Leslie GD. Nurse resilience: a concept analysis. Int J Ment Health Nurs. 2020; 29(4): 553-75. Available from: https://doi.org/10.1111/inm.12721

10. Ford S. Exclusive: Nursing Times survey reveals negative impact of Covid-19 on nurse mental health. Nursing Times. 2020. [cited 2020 May 30] Available from: https://www.nursingtimes.net/ news/mental-health/exclusive-survey-reveals-negative-impactof-covid-19-on-nurse-mental-health-29-04-2020
11. Hackett K. COVID-19: redeployment training is non-existent or inadequate, nurses reveal. Nursing Standard. 2020. [cited 2020 May 30] Available from: https://rcni.com/nursing-standard/ newsroom/news/covid-19-redeployment-training-non-existentor-inadequate-nurses-reveal-160301

12. National Nurses United. NNU COVID-19 Survey Results - 12 May 2020. National Nurses United. 2020. [cited 2020 May 30] Available from: https://www.nationalnursesunited.org/covid-19survey

13. Kaplan LS, Kleinpell R, Maves R, Doersam JK, Raman R, Ferraro DM. Critical care clinician reports on coronavirus disease 2019: results from a national survey of 4,875 ICU providers. Crit Care Expl. 2020; 2: e0125. Available from: https://doi.org/10.1097/ CCE.0000000000000125

14. Bairin P, Berteau B. France to give bonus to healthcare workers working with Covid-19 patients. CNN. 2020. [cited 2020 May 30] Available from: https://www.cnn.com/ asia/live-news/coronavirus-pandemic-intl-04-15-20/h ba1eed6bac2ac64249e730ef7f903674

15. France 24. Covid-19: France's health workers to get 'significant' pay rises. France 24. 2020. [cited 2020 May 30] Available from: https://www.france24.com/en/20200525-france-s-healthworkers-to-get-significant-pay-rises-after-coronavirus-pandemic

16. Global Preparedness Monitoring Board. A world at risk: annual report on global preparedness for health emergencies. World Health Organization. 2019. [cited 2020 May 30] Available from: https://apps.who.int/gpmb/assets/annual report/GPMB annualreport 2019.pdf

17. Roy Morgan. ABC most trusted | Facebook most distrusted. Roy Morgan. 2016. [cited 2020 May 30] Available from: http://www.roymorgan.com/findings/7641-media-net-trustjune-2018-201806260239

18. American Press Institute. A new understanding: what makes people trust and rely on news. American Press Institute. 2016. [cited 2020 May 30] Available from: https://www.americanpressinstitute.org/publications/reports/ survey-research/trust-news/single-page/

19. Abuhammad S, AlAzzam M, Mukattash T. The perception of nurses towards their roles during the COVID-19 pandemic. Int J Clin Prac. 2021; 75: e13919. Available from: https://doi.org/10.1111/ijcp.13919 